\title{
Inhaler de la fumée provoque l'inhalation de fumée: mets ça dans ton narguilé!
}

\author{
Marco L.A. Sivilotti, MD, MSc; Riyad B. Abu-Laban, MD, MHSc ${ }^{\dagger}$
}

Les urgentistes n'ont pas besoin de se faire rappeler d'être vigilants en ce qui a trait aux intoxications accidentelles au monoxyde de carbone. Parce que ses symptômes précoces sont vagues et ressemblent à ceux d'une maladie d'origine virale, on appelle le monoxyde de carbone un « grand mystificateur ». Un diagnostic erroné met les patients à risque d'une exposition prolongée s'ils retournent dans le même environnement; de plus, cela représente une occasion manquée d'identifier d'autres victimes. Dans ce numéro, La Fauci et collaborateurs nous mettent en garde contre une cause inhabituelle d'intoxication au monoxyde de carbone: fumer le narguilé, aussi appelé pipe à eau ou houka. ${ }^{1}$ Les rapports de cas ne servent pas seulement à nous avertir d'être vigilants, mais également à identifier et à nous prévenir de l'approche de quelque chose d'important. Fumer le narguilé devient de plus en plus populaire au Canada, et, selon les signalements obtenus des intéressés dans un sondage récent, sa prévalence a atteint le quart des jeunes adultes. ${ }^{2}$

L'histoire du narguilé remonte à quelque 500 ans en arrière, en Asie du Sud et au Moyen-Orient, où son emploi demeure généralisé. Au cours des dernières années, des cafés narguilé sont apparus dans les villes canadiennes et on peut acheter des pipes et fournitures pour narguilé ouvertement en magasin ou sur Internet. La plupart des fumeurs de narguilé canadiens sont des jeunes des zones urbaines qui fument du tabac ou d'autres produits végétaux parfumés avec des plantes aromatiques. Au-delà de l'inquiétude légitime concernant les effets chroniques de l'emploi du narguilé sur la santé, le cas présenté par La Fauci et collaborateurs met en évidence le risque, en une seule séance, d'une intoxication aiguë causée par ce grand mystificateur qu'est le monoxyde de carbone.
Il existe une distinction importante entre fumer le narguilé et les autres méthodes de fumer. Contrairement aux cigarettes ou à la pipe, la principale source de combustion d'un narguilé est le charbon de bois, qui est séparé du shisha (tabac ou autre végétal que l'on fume) par une plaque perforée. En conséquence, les utilisateurs inhalent principalement la fumée du charbon de bois lui-même lorsqu'ils aspirent à partir de l'embout. En fait, l'essentiel du monoxyde de carbone trouvé dans la fumée d'un narguilé provient du charbon de bois plutôt que du shisha. Du point de vue du risque lié au monoxyde de carbone, peu importe que le shisha contienne du tabac, du cannabis, des herbes aromatiques, ou même des aiguilles de pin. En effet, fumer un narguilé s'apparente beaucoup à respirer la fumée d'un feu de charbon. Bien que l'eau du siphon rafraîchisse et humidifie la fumée, elle ne filtre pas le monoxyde de carbone, lequel est peu soluble dans l'eau. Le fait de refroidir et d'humidifier la fumée peut même augmenter la dose de monoxyde de carbone inhalée en permettant aux utilisateurs de prendre des inspirations beaucoup plus longues et de tolérer des séances de fumée plus longues- 3 heures pour le rapport de cas actuel. ${ }^{3}$ Les arômes ou herbes aromatiques ajoutés à plusieurs types de shisha peuvent également donner l'illusion d'une fumée non toxique ou même bénéfique et peuvent augmenter l'attrait du narguilé chez les jeunes ou chez les fumeurs inexpérimentés. ${ }^{4}$

La concentration de monoxyde de carbone trouvée dans les fumées d'un narguilé varie, mais elle augmente lorsque la combustion est incomplète, comme cela peut survenir si le pare-vent est mal installé ou si la configuration du charbon de bois en combustion n'est pas optimale. ${ }^{5}$ Même dans des conditions optimales, cette concentration de

Service de médecine d'urgence, Université Queen's, Kingston (Ontario), et service de médecine d'urgence, Vancouver General Hospital, Vancouver (C.-B.)

Demandes : Dr Marco L. A. Sivilotti, service de médecine d'urgence, Université Queen's, 76 rue Stuart, Kingston (Ontario) K7L 2V7; marco. sivilotti@queensu.ca.

L'article a été évalué par les pairs.

(c) Canadian Association of Emergency Physicians 
monoxyde de carbone excède de beaucoup celle qui est évacuée par l'échappement de la Toyota 4-Runner 2000 conduite par l'un d'entre nous. Dans le sang, les niveaux de carboxyhémoglobine mesurés sont beaucoup plus élevés chez les fumeurs de narguilé que chez les fumeurs de cigarettes. ${ }^{6}$ Dans une étude portant sur des sujets humains volontaires, les concentrations de carboxyhémoglobine ont excédé $20 \%$, après 30 minutes, chez 3 des 45 sujets auxquels on avait dit de « fumer à leur rythme et de leur façon habituelle $\gg .7$ Dans une autre étude réalisée dans un café narguilé, les clients ont inhalé, en moyenne, $130 \pm 21 \mathrm{~L}$ de fumée contenant $150 \pm 26 \mathrm{mg}$ de monoxyde de carbone en 1 heure. Précisons ici qu'une dose de $600 \mathrm{mg}$ de monoxyde de carbone suffit à lier la moitié de l'hémoglobine totale d'un adulte. Les fumeurs de narguilé qui habitent au pied des montagnes ou en régions montagneuses doivent aussi tenir compte du fait que l'altitude, même modérée, augmente la toxicité du monoxyde de carbone.

Le fait de fumer à l'extérieur ne rend pas le narguilé moins dangereux. La séance décrite par La Fauci et collaborateurs s'est déroulée sur une plage, en terrain découvert, et non pas dans une pièce minuscule remplie de fumée. Il est également important de noter que la patiente était une adolescente. Comme plus de jeunes Canadiennes fument le narguilé, le risque d'une exposition au monoxyde de carbone pendant la grossesse augmente. Le foetus se développe dans un environnement faible en oxygène en aval du placenta et est extrêmement sensible à la présence de monoxyde de carbone, même à de très faibles concentrations. En outre, aucun des compagnons fumeurs de la patiente ne s'est présenté pour une évaluation, une situation malheureusement souvent observée lorsqu'il est question d'exposition au monoxyde de carbone.

La prévention des intoxications sera le thème de la semaine SécuriJeunes (SafeKids Week), en mars 2012, un événement qui mettra l'accent sur la santé publique, la législation, et les études. Il est clair que de fumer le narguilé peut causer une intoxication aiguë au monoxyde de carbone. Ne serait-il pas paradoxal que nos jeunes adoptent le narguilé en partie à cause de la condamnation de la cigarette? ${ }^{74}$ Compte tenu du sain respect que nos urgentistes portent au monoxyde de carbone, ce cas présenté par La Fauci et collaborateurs soulève plusieurs questions importantes concernant la prévention des accidents. Les professionnels de la médecine d'urgence au Canada devraient-ils se joindre au débat sur la légalité des cafés narguilé? À ce jour, ce débat a principalement porté sur l'applicabilité des règlements municipaux et sur la toxicité chronique de la fumée du tabac. ${ }^{8}$ Devrions-nous nous joindre à ceux qui exigent d'étendre l'interdiction de fumer aux parcs publics et espaces extérieurs, afin de « dénormaliser » encore davantage le tabagisme face aux enfants, qui sont impressionnables?? Devrions-nous attendre de Santé Canada qu'elle étudie la possibilité d'interdire le narguilé aux termes de la Loi canadienne sur la sécurité des produits de consommation, une nouvelle loi destinée à protéger le public contre les produits dangereux pour la santé par suite de leur utilisation normale? Ou devrions-nous simplement nous contenter d'informer nos patients et collaborateurs afin qu'ils soient « conscients » de ce qui semble évident: qu'inhaler de la fumée ou brûler du charbon de bois à l'intérieur peut être une mauvaise idée? Ni les usages traditionnels ni les tendances populaires chez les jeunes ne devraient être exempts de contrôles et du gros bon sens.

Conflit d'intérêts: Au moins un des auteurs signale avoir fumé du tabac avec un narguilé au cours de ses voyages et lors de son mariage; il n'a toutefois reçu aucune compensation à cet effet, et a souvent souffert de maux de tête liés à cette activité.

Mots clés: fumer, houka, monoxyde de carbone, narguilé, pipe à eau

\section{RÉFÉRENCES}

1. La Fauci G, Weiser G, Steiner IP, Shavit I. Carbon monoxide poisoning in narghile (water pipe) tobacco smokers. CFEM 2012;15:00-00.

2. Dugas E, Tremblay ML, Low NCP, et coll. Water-pipe smoking among North American youths. Pediatrics 2010;125: 1184-9.

3. Cobb CO, Shihadeh A, Weaver MF, Eissenberg T. Waterpipe tobacco smoking and cigarette smoking: a direct comparison of toxicant exposure and subjective effects. Nicotine Tob Res 2011;13:78-87.

4. Roskin J, Aveyard P. Canadian and English students' beliefs about waterpipe smoking: a qualitative study. BMC Public Health 2009;9:10.

5. Sajid KM, Akhter M, Malik GQ. Carbon monoxide fractions in cigarette and hookah (hubble bubble) smoke. 7 Pak Med Assoc 1993;43:179-82.

6. Zahran FM, Ardawi MS, Al Fayez SF. Carboxyhemoglobin concentrations in smokers of sheesha and cigarettes in Saudi Arabia. Br Med 7 (Clin Res Ed) 1985;291:1768-70.

7. Hakim FM, Hellou EB, Goldbart AM, et al. The acute effects of water-pipe smoking on the cardiorespiratory system. Chest 2011;139:775-81.

8. CBC Radio. Q. Should hookah be exempt from anti-smoking rules? Available at: http://www.cbc.ca/q/blog/2011/09/06/ should-hookah-be-exempt-from-anti-smoking-rules/ (accessed October 15, 2011).

9. Colgrove J, Bayer R, Bachynski KE. Nowhere left to hide? The banishment of smoking from public spaces. $N$ Engl 7 Med 2011;364:2375-7. 